\title{
Predisposición desfavorable hacia el aprendizaje de las Matemáticas: una propuesta para su medición*
}

\author{
Unfavorable predisposition towards mathematics learning: a measurement proposal \\ Predisposição desfavorável para a aprendizagem das Matemáticas: \\ uma proposta para sua medição
Gamal Cerda ${ }^{1}$, Rosario Ortega Ruiz', José Antonio Casas ${ }^{3}$, Rosario del Rey ${ }^{4}$ Carlos Pérez ${ }^{5}$ \\ ${ }^{1}$ Facultad de Educación, Universidad de Concepción, gamal.cerda@udec.cl \\ ${ }^{2}$ Universidad de Córdoba; Laboratorio de Estudios sobre Convivencia y Prevención de la Violencia, \\ LAECOVI (www.uco.es/laecovi) \\ ${ }^{3}$ Universidad Católica San Antonio, Murcia; LAECOVI \\ ${ }^{4}$ Departamento de Psicología Evolutiva y de la Educación, Universidad de Sevilla; LAECOVI \\ ${ }^{5}$ Facultad de Ciencias Físicas y Matemáticas, Universidad de Concepción
}

\section{RESUMEN}

Este estudio presenta la utilización y adaptación de una escala para evaluar las actitudes, emociones y creencias de los estudiantes sobre su competencia en matemáticas, así como también la autovaloración respecto de su éxito/fracaso académico en la disciplina curricular de Matemáticas. La muestra está compuesta por n= 1154 estudiantes chilenos de enseñanza secundaria. Los resultados dan cuenta de la capacidad del factor de bloqueo emocional ante las tareas matemáticas como dimensión explicativa sobre el éxito o fracaso ante dichas tareas, pero no confirman ni la dimensión de autoconfianza ni la llamada resiliencia, como factores relevantes para dicha explicación. Los resultados se discuten en relación con la literatura sobre el papel de las actitudes y predisposición ante el aprendizaje de esta disciplina curricular. Además, se discute también la necesidad educativa de prestar atención a los factores emocionales en esta área del curriculum escolar de la Educación chilena.

Palabras clave: predisposición, matemáticas, análisis factorial.

\section{ABSTRACT}

This study presents the use and adaptation of a scale that assesses both students' personal attitudes, emotions and beliefs regarding their mathematics competencies as well as students' self-appraisal regarding their success or academic failure in Mathematics as a curriculum discipline. The sample is composed of $n=1154$ secondary Chilean students. The results show that the factor "emotional block" is a relevant explanatory dimension regarding success or failure in mathematical tasks; on the other hand, the dimensions self-confidence and resilience were not found to be explanatory factors for success or failure in mathematics. The results are discussed in relation to the literature on the role of attitude and predisposition towards learning for this curriculum discipline. Additionally, the educational need to pay attention to emotional factors in this area of the Chilean educational curriculum is also discussed in this article.

Keywords: predisposition, mathematics, factor analysis.

Trabajo derivado del Proyecto Regular de Investigación FONDECYT No 1130519, financiado por CONICYT. Se agradece el financiamiento otorgado por el Proyecto Basal FB0003 del Programa de Investigación asociativa de CONICYT. 


\section{RESUMO}

Apresenta-se a utilização e adaptação de uma escala para avaliar atitudes, emoções e crenças dos estudantes sobre competência deles em matemática, assim como a autovalorização relativa ao sucesso/fracasso acadêmico nessa disciplina curricular. A mostra está composta por $\mathrm{n}=1154$ estudantes chilenos de ensino secundário. Resultados dão conta de que a capacidade do fator de bloqueio emocional diante das tarefas matemáticas como dimensão explicativa sobre o êxito ou fracasso diante das tarefas, não confirmam nem a dimensão da autoconfiança nem da chamada resiliência. Resultados são discutidos em relação à literatura sobre o papel das atitudes e predisposição diante da aprendizagem desta disciplina curricular. Além disso, discute-se, também, a necessidade educativa de prestar atenção em fatores emocionais nesta área do currículo escolar na Educação chilena.

Palavras chave: predisposição, matemáticas, análise fatorial.

\section{INTRODUCCIÓN}

La matemática es una asignatura o disciplina escolar hacia la que muchos estudiantes manifiestan una predisposición de carácter negativo o incluso rechazo por diversos motivos, como pueden ser el método utilizado, las expectativas y estilo del profesor, la influencia de los estereotipos basados en factores sociales y culturales (Guven \& Cabakcor, 2013; Moenikia \& Zahed-Babelan, 2010; Yaratan \& Kasapoğlu, 2012). Pero quizás también deberíamos incluir las propias creencias del estudiante sobre sus expectativas de éxito, su percepción de autoeficacia (Hailikari, Nevgi \& Komulainen, 2008; Nosek \& Smyth, 2011; Selkirk, Bouchey \& Eccles, 2011; Vandecandelaere et al., 2012), estas actitudes y creencias desfavorables vinculándose fuertemente al fracaso académico (Akin \& Kurbanoglu, 2011; Parker et al., 2013; Samuelsson \& Granstrom, 2007; Yaratan \& Kasapoğlu, 2012). Consecuentemente con estos estudios, se ha hallado una menor motivación para el estudio de unos contenidos que resultan imprescindibles para la futura formación intelectual de los escolares (Nasiriyan et al., 2011).

Son varias las investigaciones donde se ha puesto de manifiesto que la motivación y algunas emociones podrían estar favoreciendo o dificultando las actitudes ante los aprendizajes de las matemáticas (Núñez-Peña, Suárez-Pellicioni \& Bono, 2013; Schweinle, Meyer \& Turner, 2006; Zan et al., 2006). Y de forma más concreta, existen estudios que han vinculado emociones, como la ansiedad y el bloqueo, al rendimiento o logro académico en esta disciplina. Pekrun, Elliot y Maier, (2009) encontraron que existen ciertas emociones, tanto positivas como negativas, que se activan en los procesos de aprendizaje. Estas juegan un relevante papel en las tareas cognitivas que intervienen en los aprendizajes escolares, destacando entre otras el goce, la curiosidad, la satisfacción, el aburrimiento, la ira, la esperanza, el orgullo, la ansiedad, la desesperanza y la vergüenza. Dichos matices emocionales pueden estar actuando en tanto que mediadores de las relaciones entre las metas de logro y el nivel de rendimiento en matemáticas, señalando que ejercen un impacto negativo importante en procesos de desajuste académico y dando lugar a fracasos escolares (Moenikia \& Zahed-Babelan, 2010; Morony et al., 2013; Rosário et al., 2012; Suárez-Álvarez, Fernández \& Muñiz, 2013; Yaratan \& Kasapoğlu, 2012). Igualmente se ha señalado el efecto positivo que la buena predisposición hacia las tareas matemáticas tiene respecto de la reducción de la ansiedad ante estos aprendizajes (Akin y Kurbanoglu, 2011). Otros trabajos al respecto han encontrado que los estudiantes con mejores actitudes hacia las matemáticas tienen más altas percepciones de utilidad de las mismas y denotan motivaciones intrínsecas relacionadas con su estudio (Perry, 2011). Hidalgo, Maroto, y 
Palacios (2005), y McLeod (1992), encontraron que una mayor confianza en el aprendizaje de esta disciplina se correspondía con un mayor éxito en la meta de su dominio. Fennema y Sherman (1976) habían encontrado que conductas de acercamiento a estas tareas resultaban positivas en el éxito de los aprendizajes de esta disciplina. Miñano y Castejón (2011); Sakiz, Pape, y Hoy (2012), encontraron que una actitud positiva hacia el aprendizaje de las matemáticas incidía en el rendimiento escolar en esta disciplina.

Estos estudios se han desarrollado paralelamente al diseño e implementación de instrumentos de medida, que permitan discriminar entre unos factores y otros en el proceso de adquisición de dominio y competencia ejecutiva en este ámbito curricular, especialmente aquellos del ámbito afectivo o actitudinal, donde se pueden mencionar varios instrumentos que intentan su medida (Chamberlin, 2010). Existen varias escalas que examinan las actitudes hacia las matemáticas, tales como la escala de Fennema y Sherman (1976), adaptada y validada en diferentes países y situaciones escolares, y que examina nueve dimensiones que recogen información acerca de la actitud hacia el éxito en matemáticas, el rol de padres y maestros, la confianza en el aprendizaje, la ansiedad y motivación, entre otras. O también, The Attitude Toward Mathematics Inventory (ATMI), de Tapia y Marsh (2004), que evalúa seis dimensiones respecto de este constructo, a saber: Confianza-auto-concepto, Ansiedad, Utilidad-valor de las matemáticas, Gusto por las matemáticas, Motivación y Expectativa de los padres y profesores. La mayor parte de estos instrumentos se han desarrollado en escenarios escolares de secundaria (Muñoz y Mato, 2008; Alemany y Lara, 2010). Una excepción, en este sentido, es el trabajo de Adelson y McCoach (2011), quienes elaboraron una escala de actitudes hacia las matemáticas para estudiantes de primaria que examina sólo dos factores: la percepción de eficacia y el gusto por las matemáticas.

Los estudios previos adolecen de un enunciado teórico que permita interpretar el significado de cada uno de los factores que marcan las relaciones entre las actitudes, predisposiciones, creencias y emociones que se estimulan en el aprendizaje escolar, y el éxito o fracaso en el dominio de ciertos contenidos curriculares concretos, como es el caso de las matemáticas.

Por nuestra parte, hemos puesto la confianza en el modelo teórico de Claxton (2001), en el que se enuncia un heurístico que relaciona las creencias y autoreferencias hacia la propia competencia, con valoraciones positivas o negativas, como motor del logro o como causa del fracaso en el éxito del dominio y competencia en determinada materia del curriculum escolar. Tal aproximación teórica, es también conocida como teoría de las tres $\mathrm{R}$ (reflexión, recursos y resiliencia): reflexión, percepción de la competencia para afrontar un conocimiento nuevo de forma reflexiva; recursos, o percepción de que cuando algo no se domina se deben buscar nuevos recursos para afrontar la dificultad; y resiliencia, o capacidad para resistir la frustración y los efectos del fracaso real o imaginado. El bloqueo emocional que dificulta de reflexión y el acceso a los recursos (ansiedad), y la debilidad de la competencia para la resiliencia, como mecanismo para la reestructuración del equilibrio emocional ante un fracaso o trauma, juegan un papel relevante en las dificultades en cualquier proceso de aprendizaje que exija la autodirección de las tareas cognitivas implícitas. Esta es una de las escasas investigaciones que introduce matices actitudinales o de predisposición negativa ante el aprendizaje de las matemáticas.

Por nuestra parte, y a partir del modelo teórico que realiza Claxton, hemos tratado de comprobar, validando un instrumento previo, hasta qué punto la autoconciencia de tener o no una cierta disposición, positiva y negativa, hacia el aprendizaje de las matemáticas, tiene relevancia en términos de éxito o fracaso en estas tareas curriculares. Es esta la finalidad 
de este estudio, además de validar en una población de escolares chilenos el instrumento diseñado por Madera, Ortega-Ruiz y Del Rey (2009) de medida de las actitudes positivas (autoconfianza y resiliencia) y negativas (bloqueo emocional y miedo al fracaso). Dicho instrumento había sido validado para una pequeña muestra de escolares españoles (Del Rey, Madera y Ortega-Ruiz, 2011). Nuestro objetivo, a este respecto busca encontrar si se mantiene o no la solución factorial original y de no ser así, cuál es la que más se ajusta a la población de estudiantes chilenos. Una vez realizado dicho proceso de validación, hemos buscado encontrar la importancia que la predisposición y actitudes ante las Matemáticas tienen como factor de éxito o fracaso en esta tarea curricular.

\section{MÉTODO}

\subsection{PARTICIPANTES}

En este estudio participaron 1.154 estudiantes de educación media, de primero a cuarto año. Con edades comprendidas entre los 13 y los 20 años $(M=15.96 ; D T=1.28)$, el 52,9\% de los estudiantes son mujeres.

\subsection{INSTRUMENTO}

Se utilizó el cuestionario tipo Likert de 13 ítems (Del Rey, Madera y Ortega, 2011), donde existen 3 dimensiones. La primera de ellas aborda el bloqueo emocional ante el aprendizaje de las matemáticas. Propuesta por las autoras, ésta examina la percepción del estudiante respecto a sus malos resultados en la materia; su eventual incapacidad para resolver problemas o su falta de gusto por la asignatura. Está constituida por 5 ítems: "En matemáticas sé que no voy a tener éxito"; "Mis resultados en matemáticas siempre han sido malos"; "No sirvo para las matemáticas"; "Las matemáticas no me gustan" y "Nunca me salen los problemas" $\left(r_{\alpha}=.83\right)$. La segunda dimensión, la de resiliencia ante el aprendizaje de las matemáticas, examina la persistencia a la hora de resolver problemas matemáticos; la satisfacción ante el trabajo bien hecho; el esfuerzo ante la tarea; y está constituida por 3 ítems: "Cuando un problema no me sale por un método, voy probando otras cosas"; "Cuando un problema me sale bien, me siento contento" y "Me esfuerzo mucho para entender las matemáticas" $\left(r_{\alpha}=.64\right)$. Finalmente, la dimensión de autoconfianza, examina la confianza del alumnado en su memoria para resolver problemas; la no necesidad de estudiar; la capacidad de indagación; la diversión y la facilidad para comprender la materia, entre otras cosas. Ésta está constituida por 5 ítems: "Para tener éxito en matemáticas no tengo que estudiar"; "La memoria me sirve para resolver problemas matemáticos"; "Cuando un problema no me sale por un método, voy probando otras cosas"; "Me gusta "jugar" con los números y pensar en ellos" y "Las operaciones con números me resultan fáciles" $\left(r_{\alpha}=.67\right)$.

$\mathrm{Al}$ ser una escala que da cuenta de la percepción de los estudiantes, sobre cuestiones subjetivas, con connotaciones afectivas y valorativas, la escala tipo Likert resulta ser una excelente modalidad de pesquisa, pues el estudiante responde mostrando su grado de acuerdo con el enunciado desde 1 (totalmente en desacuerdo) hasta 5 (totalmente de acuerdo), lo que aporta la flexibilidad necesaria en las respuestas para el análisis de los resultados (Muñoz y Mato, 2008). 


\subsection{PROCEDIMIENTO Y ANÁLISIS DE DATOS}

Para analizar la idoneidad de la solución factorial de la escala original, se realizaron análisis factoriales exploratorios (AFE) y, posteriormente, análisis factoriales confirmatorios (AFC). Para dichos análisis, fueron utilizados los software estadísticos Factor 9.2 (LorenzoSeva \& Ferrando, 2006) y EQS 6.2 (Bentler, 1995), respectivamente. Para la idoneidad del ajuste del AFC se ha tenido en cuenta, siguiendo a Hu \& Bentler (1999), los índices RMSEA (Root Mean Square Error of Approximation), cuyos valores recomendables son $<.08$; y los índices NNFI (Non-Normed Fit Index), IFI (Incremental Fit Index), y CFI (Comparative Fit Index), cuyos valores recomendables son $>.90$

\section{RESULTADOS}

En primer lugar, se ha realizado un AFE mediante el método de estimación de máxima verosimilitud robusta (RML), a través del programa Factor, que permite realizar este tipo de análisis con matrices policóricas y estimadores adecuados. Esto, debido a la naturaleza ordinal de las variables y a su distribución multivariante no normal, como indica el coeficiente de Mardia (Mardia, 1970), con un valor de 232.51. Se empleó la rotación Direct Oblimin y el procedimiento para determinar el número de dimensiones Hull (Lorenzo-Seva, Timmerman, \& Kiers, 2011). Todo ello adecuado a la no normalidad de la muestra y el carácter ordinal de las variables, mostrando una adecuación de la matriz de correlaciones policóricas adecuado, con un valor Kaiser-Meyer-Olkin $(K M O)$ test $=.87$, y de la prueba de esfericidad de Barlett's $=3734.0(d f=78 ; p=.00)$.

La solución factorial obtenida muestra 3 factores que explican un $56 \%$ de la varianza total. Sin embargo, como se puede observar en la Tabla 1, la solución rotada muestra grandes diferencias con la solución original de la escala en la población española y la propuesta del constructo teórico de las autoras.

Tabla 1. Solución factorial inicial AFE (valores omitidos menores de 0.300)

\begin{tabular}{|c|c|c|c|}
\hline Ítem & Factor 1 & Factor 2 & Factor 3 \\
\hline 1 & & 0.730 & \\
\hline 2 & & -0.317 & 0.501 \\
\hline 3 & & -0.370 & -0.318 \\
\hline 4 & & 0.740 & \\
\hline 5 & & & \\
\hline 6 & & 0.859 & \\
\hline 7 & & & 0.526 \\
\hline 8 & & & 0.500 \\
\hline 9 & & 0.753 & \\
\hline 10 & 0.474 & -0.315 & \\
\hline 11 & & 0.699 & \\
\hline 12 & & 0.341 & 0.395 \\
\hline 13 & & -.631 & \\
\hline
\end{tabular}




\subsection{ANÁLISIS FACTORIAL CONFIRMATORIO}

Posteriormente, se realizó un AFC, donde se intentó de nuevo corroborar la solución factorial de tres factores original (ver figura 1), obteniendo un ajuste poco aceptable con valores de: $\chi^{2 \mathrm{~S}-\mathrm{B}}=473.45(p=.00) ; \mathrm{RMSEA}=.10 ; \mathrm{CFI}=0.89 ; \mathrm{NNFI}=.88$ y $\mathrm{IFI}=.91$; esto, además con unos pesos factoriales (ver figura 1) nada aceptables en las dimensiones de autoconfianza y resiliencia, todos inferiores a .50 exceptuando el ítem 13.

Figura 1. Solución Factorial de 3 factores en población chilena.

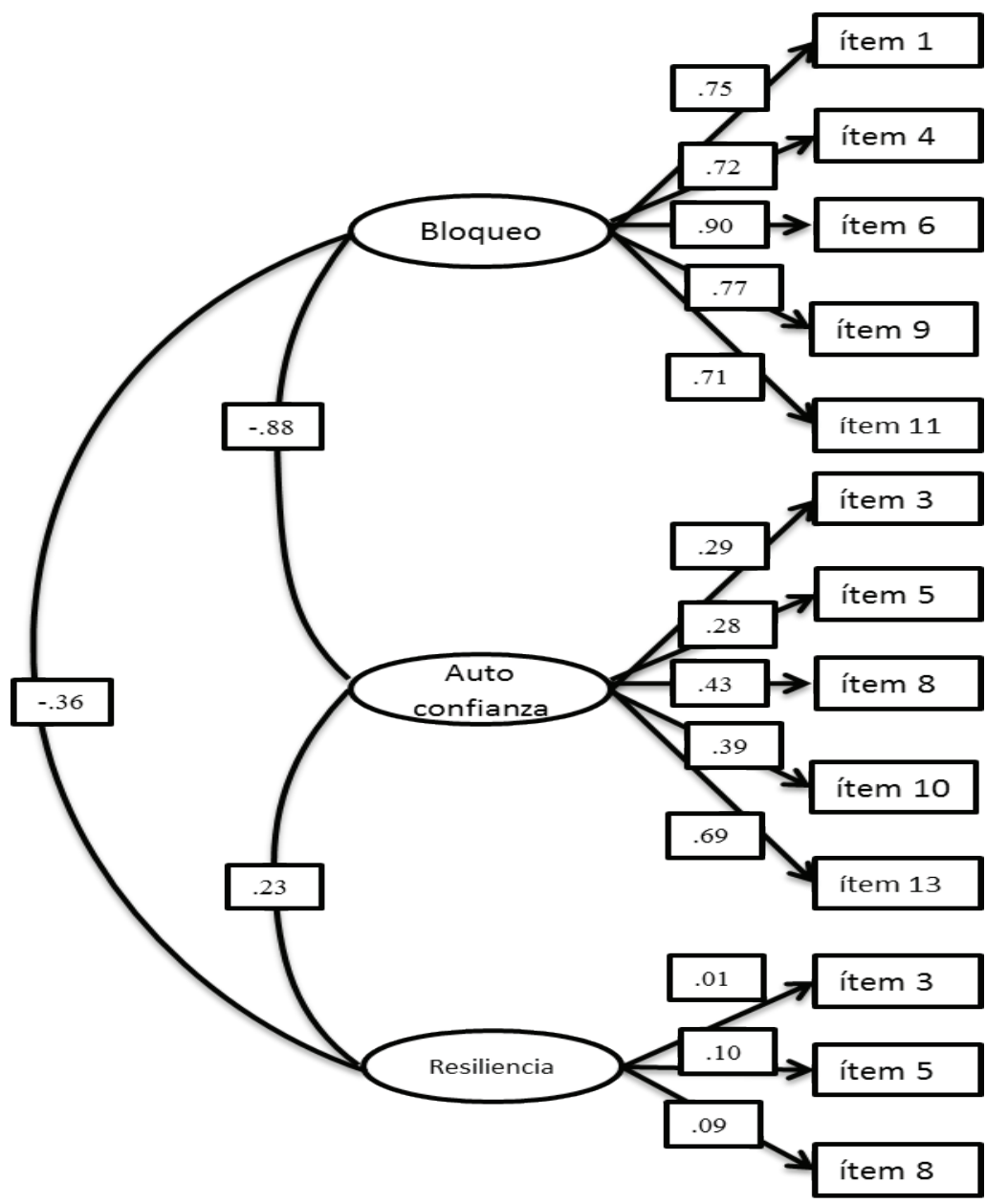

Tras estos resultados se decidió analizar, entonces, las soluciones factoriales de cada una de las 3 dimensiones de la escala para observar sus propiedades psicométricas. 
Tabla 2. Soluciones Factoriales para las 3 dimensiones postuladas (AFE)

\begin{tabular}{|l|c|c|c|c|c|}
\hline \multicolumn{1}{|c|}{ Factor } & $\mathrm{c} 2(\mathrm{gl})$ & CFI & TLI & RMSEA & SRMR \\
\hline Factor 1 Resiliencia & $315,78 * * *(32)$ & .82 & .85 & .08 & .08 \\
\hline Factor 2 Auto Confianza & $148,77 * * *(29)$ & .89 & .90 & .07 & .06 \\
\hline Factor 3 Bloqueo & $136.69 * * *(20)$ & .95 & .96 & .04 & .03 \\
\hline
\end{tabular}

$* * *: \mathrm{p}<0,001$

Como se observa en la Tabla 2, de los tres modelos factoriales analizados, sólo el tercer factor, o tercera dimensión, mostró un adecuado ajuste a los datos, toda vez que presentó valores en los coeficientes CFI y TLI cercanos al valor de referencia de 0,95 , señalado como indicador de un buen ajuste. A la misma conclusión se llega al analizar los valores de los coeficientes RMSEA y SRMR.

Dados los resultados anteriores, se decidió utilizar la escala unidimensional que sólo midiera el factor de bloqueo o rechazo ante las matemáticas. Los estadísticos de calidad de ajuste para la solución de un factor correspondieron a $\mathrm{c}^{2}(27)=92,17, \mathrm{CFI}=0,96$, TLI $=$ $0,95, \mathrm{RMSEA}=0,06$ y SRMR $=0,04$, lo cual indica un adecuado ajuste.

Sin embargo, para evitar problemas de aquiescencia al utilizar una escala tan pequeña, se decidió introducir el ítem 13 redactado en negativo, que valorara la posibilidad de que se cumplimente una pequeña escala de forma rápida y sin valor real.

Los resultados de calidad de ajuste indicaron una mejora al añadir el ítem 13, $\mathrm{c}^{2}(9)=$ $19,46, \mathrm{CFI}=0,99$, TLI $=0,99, \mathrm{RMSEA}=0,05$ y $\mathrm{SRMR}=0,02$. En cuanto a las cargas factoriales, éstas se presentan en la Tabla 3.

Tabla 3. Matriz de cargas factoriales para la solución de 1 factor.

\begin{tabular}{|c|c|}
\hline Ítem & Factor 1 \\
\hline 1 & .68 \\
\hline 4 & .70 \\
\hline 6 & .86 \\
\hline 9 & .73 \\
\hline 11 & .69 \\
\hline 13 & -.61 \\
\hline
\end{tabular}

De esta manera, como consecuencia del trabajo de adaptación, se diseñó un constructo asociado a estos seis ítems, el que se denominó Predisposición hacia las Matemáticas. Con estos antecedentes, se calculó el índice de confiabilidad de esta versión mediante el coeficiente Alpha de Cronbach, que arrojó un valor de $\left(r_{\alpha=} .82\right)$ considerado adecuado para estos efectos. 


\section{CONCLUSIONES}

Tal y como se mencionó en la introducción, son múltiples las razones que podrían explicar lo que parece ser una predisposición emocionalmente refractaria ante el aprendizaje de las matemáticas (Guven \& Cabakcor, 2013; Yaratan \& Kasapoğlu, 2012), pero entre ellos está aceptado que tiene relevancia las propias creencias del estudiante sobre sus expectativas de éxito, su percepción de autoeficacia, o la influencia de creencias sobre estereotipos sociales o culturales (Hailikari, Nevgi \& Komulainen, 2008; Nosek \& Smyth, 2011; Selkirk, Bouchey \& Eccles, 2011).

Nuestros resultados confirman que, efectivamente, algunos aspectos de estas creencias inciden de forma relevante en ello. La idea de que la dimensión de bloqueo emocional o predisposición negativa ante estas tareas es un verdadero hándicap en la percepción de fracaso en las mismas, ha sido confirmada. No cabe duda de que los estudiantes que se perciben con expectativas de fracaso ("en matemáticas sé que no voy a tener éxito"), que han registrado o que tienen una historia de fracasos ("mis resultados en matemáticas siempre han sido malos"), que nunca consiguen hacer los problemas o ejercicios de matemáticas ("las matemáticas no me gustan; "nunca me salen los problemas), están en una posición de desventaja para asumir estos aprendizajes. Se confirma, pues, que la dimensión de bloqueo emocional es potencialmente explicativa cuando se trata de comprender el fracaso en estas tareas.

No tan claro resulta, siguiendo la teoría de Claxton (2001), asegurar que la autoconfianza en la propia competencia sea un factor determinante en el éxito, e igualmente es también muy débil el factor resiliencia o capacidad de sobreponerse al fracaso, un elemento que pueda explicar gran parte de la varianza que unos y otros escolares presentan ante las tareas matemáticas.

Con este estudio, se pone de manifiesto la importancia de la predisposición negativa, pero no podemos hacer lo mismo con las otras dos dimensiones (autoconfianza y resiliencia) a la búsqueda de un modelo explicativo que confirme la teoría de la que partimos (Claxton, 2001; Madera, Ortega-Ruiz y Del Rey, 2009; Del Rey, Madera y Ortega-Ruiz, 2011).

La diferencia del tamaño de las dos muestras en las que se aplicó el instrumento, los elementos culturales y de carácter educativo, o incluso, las diferencias curriculares en la enseñanza y aprendizaje de las matemáticas, podrían estar en la base de la diferencia entre los resultados encontrados por Del Rey, Madera y Ortega (2011), y los que hemos obtenidos con estudiantes chilenos. No cabe duda de que los elementos que componen la conciencia de autoconfianza (representada en el instrumento por creencias como que para tener éxito no hay que estudiar; que jugar con los números es divertido y fácil, que sólo con la memoria se pueden resolver problemas), no se ha manifestado como una dimensión sólida y coherente que permita explicar o contribuir a un modelo explicativo sobre predisposiciones y actitudes, y éxito escolar en esta materia.

Igualmente, creencias en el propio esfuerzo, en la creatividad buscando procedimientos diversos, en la alegría ante el éxito, y otras de la misma naturaleza (que en el instrumento de origen, hemos considerado expresiones de resiliencia), tampoco tiene la solidez esperada como factor explicativo ni como factor de un modelo único. No podemos por tanto concluir que aspectos motivacionales de carácter tan subjetivo estén apoyando o no el éxito en las tareas matemáticas, como parece que encontraron Núñez-Peña, Suárez-Pellicioni \& Bono (2013); Schweinle, Meyer \& Turner (2006) y Zan, Brown, Evans \& Hannula (2006). En 
este orden de cosas, el apoyo en las tareas escolares por parte de la madre incrementa de forma significativa el nivel de competencia matemática de los niños en matemáticas, en los ciclos educativos iniciales, lo que probablemente puede erigirse como un catalizador de la motivación hacia la disciplina (Vera, Gomzález y Hernández, 2014).

Sí confirma nuestro estudio aquellos, como los de Pekrun, Elliot y Maier (2009), que encontraron que emociones como la ansiedad y el bloqueo al rendimiento, o logro académico en esta disciplina, afectan la percepción de fracaso y pueden convertirse en hándicap para el avance en esta área del curriculum escolar, actuando como mediadores de las relaciones entre las metas de logro y el nivel de rendimiento en matemáticas. Esto interesa de sobremanera, cuando estudios en primer año de universidad revelan que el desempeño en la prueba de selección universitaria en matemáticas es un indicador relevante a la hora de explicar el rendimiento académico general observado (Barahona, 2014).

Así, estos resultados destacan una dimensión, como la de la predisposición negativa ante las matemáticas, que supera las barreras culturales y muestra buenos valores de ajuste psicométrico, frente a las dos dimensiones de resiliencia y autoconfianza, que no se ajustan a estas condiciones. De esta manera, en futuras investigaciones, la importancia de esta dimensión sugiere la posibilidad de emplear los ítems de esta dimensión como una escala unidimensional enfocada a la evaluación en exclusiva de este bloqueo o predisposición negativa ante las matemáticas. Se trata de una pequeña escala de 6 ítems, que recoge igualmente un ítem redactado en negativo para controlar la aquiescencia (tendencia a responder afirmativamente independientemente del contenido por el que se pregunta) y que muestra unos muy buenos índices de ajuste. La puntuación obtenida en la escala no sólo se ha relacionado de forma directa con el rendimiento académico de los estudiantes en matemáticas, al punto que aquellos estudiantes que puntúan alto en ella, es decir, presentan una predisposición desfavorable hacia las matemáticas, son aquellos estudiantes que tienden a presentar menores promedios de calificaciones en matemáticas, y viceversa. Además, dicha predisposición ha resultado relevante a la hora de explicar la variabilidad de los rendimientos académicos generales, y en matemáticas, de los estudiantes en modelos complejos de interacción con otras variables, como la inteligencia lógica, los esquemas de razonamiento formal, como también con el nivel de competencias matemáticas tempranas y factores de convivencia escolar (Cerda y Pérez, 2014; Cerda et al., en prensa).

Este tipo de instrumentos, con la fiabilidad y la brevedad de su uso, permiten indagar en la línea de investigación sobre la influencia de percepciones y predisposición negativa en el aprendizaje de las matemáticas. Sin embargo, la pretensión de encontrar un modelo complejo y multidimensional que articule emociones de bloqueo o negativas, como elementos de una predisposición de rechazo ante estos aprendizajes, y emociones positivas y de capacidad de resiliencia ante el fracaso en las tareas, no ha sido confirmada en este estudio. Es evidente, a partir de él que no se puede inferir desde un modelo global, como es el heurístico de la aproximación teórica de Claxton (2001), que había sido confirmado en el estudio español (Del Rey, Madera y Ortega-Ruiz), un modelo explicativo que articule tanto factores de predisposición negativa como factores de predisposición positiva y de capacidad de resiliencia. Pero sí podemos afirmar que un modelo de dimensión única (bloqueo ante las tareas matemáticas) sí permite aproximaciones explicativas. Por ello, proponemos los ítems de la dimensión de bloqueo como un instrumento útil para medir la predisposición negativa ante las matemáticas. Dicho instrumento podría denominarse Escala de Predisposición hacia las Matemáticas (EPMAT). 
Estudios Pedagógicos XLII, Nº 1: 53-63, 2016

PREDISPOSICIÓN DESFAVORABLE HACIA EL APRENDIZAJE DE LAS MATEMÁTICAS: UNA PROPUESTA PARA SU MEDICIÓN

\section{REFERENCIAS BIBLIOGRÁFICAS}

Akin, A. \& Kurbanoglu, I. (2011). The relationships between math anxiety, math attitudes, and selfefficacy: A structural equation model. Studia Psychologica, 53(3), 263-274.

Adelson, J. L. \& McCoach, D. B. (2011). Development and psychometric properties of the Math and Me Survey: Measuring third through sixth graders' attitudes towards mathematics. Measurement and Evaluation in Counseling and Development, 44, 225-247.

Alemany, I. y Lara, A. (2010). Las actitudes hacia las matemáticas en el alumnado de la ESO: un instrumento para su medición. Publicaciones, 40, 49-71.

Chamberlin, S. (2010). A review of instruments created to assess affect in mathematics. Journal of Mathematics Education, 3(1), 167-183.

Barahona, P. (2014). Factores determinantes del rendimiento académico de los estudiantes de la Universidad de Atacama. Estudios Pedagógicos, 15 (1), 25-39.

Bentler, (1995). EQS 6 Structural Equations Program Manual. Encino CA: Multivariate Software, Inc.

Cerda, G., Romera, E., Casas, J., Pérez, C. y Ortega, R. (en prensa). Influencia de variables cognitivas y motivacionales en el rendimiento académico en matemáticas en estudiantes chilenos. Educación XX1.

Cerda, G., y Pérez, C. (2014). Competencias matemáticas tempranas y actitud hacia las tareas matemáticas. Variables predictoras del rendimiento académico en educación primaria: Resultados preliminares. International Journal of Developmental and Education Psychology, 1 (7), 469-476.

Claxton, G. (2001). Aprender. El reto del aprendizaje contínuo. Barcelona: Paidós

Del Rey, R., Madera, E. \& Ortega-Ruiz, R. (2011). Validation of CAT-Ma: an instrument of measure of the emotional impact of mathematics learning. Proceedings of the $14^{\text {th }}$ Biennial conference of the European association for Research on learning and instruction, Exeter/United Kingdom.

Fennema, E., \& Sherman, J. (1976). Fennema-Sherman mathematics attitudes scales: Instruments designed to measure attitudes towards the learning of mathematics by females and males. Journal for Research in Mathematics Education, 7, 324-326.

Guven, B. \& Cabakcor, B. (2013). Factors influencing mathematical problem-solving achievement of seventh grade Turkish students. Learning and Individual Differences, 23, 131-137.

Hailikari, T., Nevgi, A. \& Komulainen, E. (2008). Academic self-beliefs and prior knowledge as predictors of student achievement in Mathematics: A structural model. Educational Psychology, 28(1), 59-71.

Hidalgo, S., Maroto, A. y Palacios, A. (2004). ¿Por qué se rechazan las matemáticas? Análisis evolutivo y multivariante de actitudes relevantes hacia las matemáticas. Revista de Educación, $334,75-95$.

Hu, L., \& Bentler, P. M. (1999). Cutoff criteria for fit indexes in covariance structure analysis: Conventional criteria versus new alternatives. Structural Equation Modeling: A Multidisciplinary Journal, 6 (1), 1-55.

Lorenzo-Seva, U. \& Ferrando, P.J. (2006). FACTOR: A computer program to fit the exploratory factor analysis model. Behavior Research Methods Instruments \& Computers, 38 (1), 88-91.

Lorenzo-Seva, U., Timmerman, M. E., \& Kiers, H.A.L. (2011). The Hull method for selecting the number of common factors. Multivariate Behavioral Research, 46, 340-364.

Madera, E., Ortega Ruiz, R., \& Del Rey, R. (2009). Impacto emocional del aprendizaje de las matemáticas: Estudio en un centro público de Córdoba. Córdoba: Universidad de Córdoba

McLeod, D. B. (1992). Resarch on affect in mathematics education: A reconceptualization. En D. A. Grouws (Ed.), Handbook of Research on Mathematics Teaching and Learning. New York: McMillan, pp. 575-596.

Miñano, P., \& Castejón, J. L. (2011). Variables cognitivas y motivacionales en el rendimiento académico en Lengua y Matemáticas: un modelo estructural. Revista de Psicodidáctica, 16 (2), 
203-230.

Moenikia, M. \& Zahed-Babelan, A. (2010). A study of simple and multiple relations between mathematics attitude, academic motivation and intelligence quotient with mathematics achievement. Social and Behavioral Sciences, 2, 1537-1542.

Morony, S., Kleitman, S., Ping Lee, Y. \& Stankov, L. (2013). Predicting achievement: Confidence vs self-efficacy, anxiety, and self-concept in Confucian and European countries. International Journal of Educational Research, 58, 79-96.

Muñoz, J. M. y Mato, M. D. (2008). Análisis de las actitudes respecto a las matemáticas en alumnos de ESO. Revista de Investigación Educativa, 26 (1), 209-226.

Nasiriyan, A. Azar, H. K. Noruzy, A. Dalvand, M. R. (2011). A model of self-efficacy, task value, achievement goals, effort and mathematics achievement. International Journal of Academic Research, 3 (2), 612-618.

Nosek, B. A. \& Smyth, F. L. (2011). Implicit social cognitions predict sex differences in math engagement and achievement. American Educational Research Journal, 48, 1124-1154.

Núñez-Peña, M., Suárez-Pellicioni, M. \& Bono, R. (2013). Effects of math anxiety on student success in higher education. International Journal of Educational Researc, 58, 36-43.

Parker, P., Marsh, H., Ciarrochia, J., Marshalla, S. \& Abduljabbarc, A. (2013). Juxtaposing math self-efficacy and self-concept as predictors of long-term achievement outcomes. Educational Psychology: An International Journal of Experimental Educational Psychology, 1-20.

Pekrun, R., Elliot, A.J., \& Maier, M.A. (2009). Achievement goals and achievement emotions: Testing of a model of their joint relations to academic performance. Journal of Educational Psychology, 101, 115-135.

Perry, C. A. (2011). Motivation and attitude of preservice elementary teachers toward mathematics. School Science and Mathematics, 111 (1), 2-10.

Rosário, P., Laurenço, A., Paiva, O., Rodrigues, A., Valle, A. y Tuero-Herrero, E. (2012). Predicción del rendimiento en matemáticas: efecto de variables personales, socioeducativas y del contexto escolar. Psicothema, 24 (2), 289-295.

Sakiz, G. Pape, S.J. \& Hoy, A.W. (2012). Does perceived teacher affective support matter for middle school students in mathematics class rooms?. Journal of school Psychology, 50, 235-255.

Samuelsson, J. \& Granström, K. (2007). Important prerequisites for students' mathematical achievement. Journal of Theory and Practice in Education, 3, 150-170.

Schweinle, A., Meyer, D. \& Turner, J. (2006). Striking the right balance: Student's motivation and affect in upper elementary mathematics classes. Journal of Educational Research, 99 (5), 271 293.

Selkirk, L., Bouchey, H., \& Eccles, J. (2011). Interactions among domain-specific expectancies, values, and gender: Predictors of test anxiety during early adolescence. Journal of Early Adolescence, 31 (3), 361-389.

Suárez-Álvarez, J. Fernández, R. \& Muñiz, J. (2013). Self-concept, motivation, expectations, and socioeconomic level as predictors of academic performance in mathematics. Learning and Individual Differences, 30, 118-123.

Tapia, M. \& Marsh, G.E. (2004). An instrument to measure mathematics attitudes. Academic Exchange Quarterly, 8 (2), 16-21.

Vandecandelaere, M., Speybroeck, S., Vanlaar, G., De Fraine, G. \& Van Damme, J. (2012). Learning environment and students' mathematics attitude. Studies in Educational Evaluation, 38, 107-120.

Vera, J., González, C. y Hernández, S. (2014). Familia y logro escolar en matemáticas del primer ciclo escolar de educación primaria en Sonora, México. Estudios Pedagógicos, 15 (1), 281-292.

Yaratan, H. \& Kasapoğlu, L. (2012). Eighth grade students' attitude, anxiety, and achievement pertaining to mathematics lessons. Social and Behavioral Sciences 46, 162-171.

Zan, R., Brown, L., Evans, J. \& Hannula, M. (2006). Affect in mathematics education: An introduction. Educational Studies in Mathematics, 63, 113-121. 
